\title{
On the Inversion of the Camber Line Problem
}

\author{
DAVID W. MIKOLAITIS*
}

P.O. Box 116250, Department of Aerospace Engineering, Mechanics \&
Engineering Science, University of Florida, Gainesville, FL 32611-6250

(Received 1 May 2000; Revised 31 May 2001)

Often airfoil shapes are given by specification of camber line and thickness functions. The camber line of an airfoil extends from the leading edge of the airfoil to the trailing edge and its position is given as a function of distance measured along the chord of the airfoil. The thickness function measures the perpendicular distance from the upper and lower surfaces of the airfoil to the camber line. The inverse problem where the upper and lower surfaces are specified and camber line and thickness functions are to be determined give rise to a first order semi-explicit nonlinear differential algebraic equation with boundary conditions. Examples are given where there are no solutions and multiple solutions.

Keywords: Differential-algebraic system; Non-linear boundary value problem; Existence; Inversion; Airfoil

AMS Classification: Primary: 34B15

\section{INTRODUCTION}

The subject of differential-algebraic equations (DAE) has seen explosive growth in recent years, especially when applied to problems in constrained rigid body dynamics and chemical process simulation. As both of these types of engineering problems typically lead to initial value conditions much of the mathematical machinery that has been developed for DAE's has been concerned with local existence and uniqueness issues and local error estimates and stability criteria for

*Tel.: 352-392-7632; Fax: 352-392-7303; E-mail: dwm@aero.ufl.edu 
various numerical procedures. An excellent reference is [1]. The problems that are the focus of this paper lead to DAE's with boundary conditions so local existence and uniqueness considerations are insufficient and non-local properties must be considered.

Here we will consider a problem motivated from the study of airfoils that naturally leads to a first order semi-explicit nonlinear differential algebraic equation with boundary conditions. The NACA four digit series of airfoils is described by a camber line function and a thickness function. The original motivation for use of the camber line holds true today. Thin airfoil theory [2, for example], which depends on knowing the camber line function, affords any easy method of calculating lifting force and moments on airfoils by performing simple quadratures. Only knowing the locations of the upper and lower surfaces poses a much more difficult computation for the lifting force and moments. The equations which describe the upper and lower surfaces given thickness and camber line functions are:

$$
\begin{aligned}
& x_{u}=x-y_{t}(x) \cdot \sin (\theta) \\
& y_{u}=y_{c}(x)+y_{t}(x) \cdot \cos (\theta) \\
& x_{l}=x+y_{t}(x) \cdot \sin (\theta) \\
& y_{l}=y_{c}(x)-y_{t}(x) \cdot \cos (\theta)
\end{aligned}
$$

where $x$ is measured along the chord from $x=0$ at the leading edge to $x=c$ at the trailing edge, $y_{t}(x)$ is the thickness function, $y_{c}(x)$ is the camber line function, and $\tan (\theta)$ is the camber line slope [3, 4]. A definition sketch is shown in Figure 1. It is not unusual to neglect the camber line slope, which simplifies the equations and makes the reverse

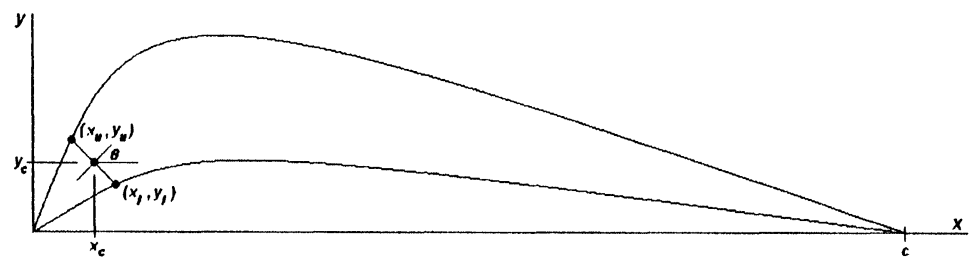

FIGURE 1 Definition sketch for airfoil coordinates. 
problem of extracting the thickness envelope and mean line for a given airfoil straightforward. Indeed, most authors use the formulation:

$$
\begin{array}{r}
y_{u}(x)=y_{c}(x)+y_{t}(x) \\
y_{l}(x)=y_{c}(x)-y_{t}(x)
\end{array}
$$

which is equivalent in the limit of nearly symmetric airfoils which have negligible camber and the inversion problem is trivial.

When the camber line slope is not neglected, which would be appropriate in situations where more exact analysis is required, the inversion problem is far from trivial in most cases and often the inversion problem has no solution. Consider, for example, the triangular region shown in Figure 2.

The camber line must start at $A$ and end at $F$. Given a point within the region $A B D$ there is only one solution to the problem of finding points on the upper surface $(A B C E F)$ and the lower surface $(A D F)$ that are equidistant from that point. The camber line must be perpendicular to the line segment that joins the points on the upper and lower surface and hence the direction of the camber line is a unique function of position within that region. Since the camber line starts at $A$ it is now clear that the camber line curve within the region $A B D$ is the bisector of the angle formed at $A$. Similarly, the camber line curve in region $D E F$ must be the bisector of the angle formed at $F$.

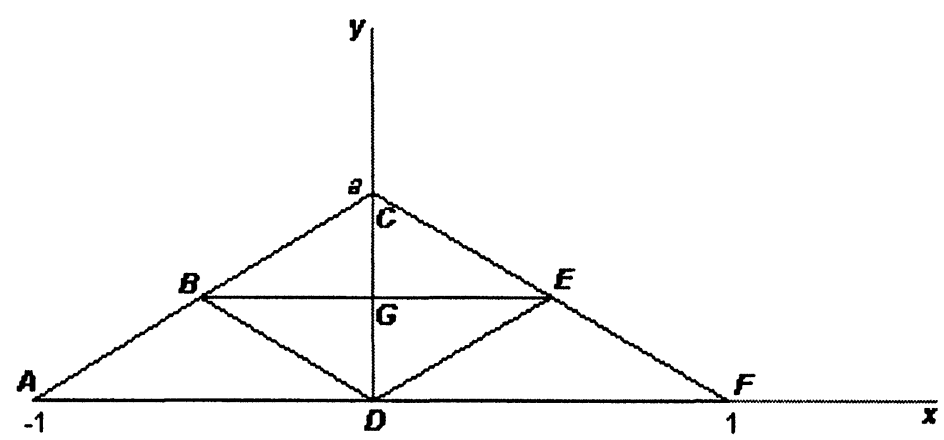

FIGURE 2 A triangular airfoil. 
Given a point within the region $B E D$ there are two distinct solutions to the problem of finding points on the upper surface $(A B C E F)$ and the lower surface $(A D F)$ that are equidistant from that point. One of the solutions has its point on the upper surface on $A B C$ and the other solution has its point on the upper surface on $C E F$. The solution curve entering $B D E$ from the left has its point on the upper surface on $A B C$ while the solution curve that leaves $B D E$ to the right has its point on the upper surface on $C E F$. The only place where the solution can smoothly change from one solution branch to the other is at the point $G$. The bisector of the angle at $A$ does not pass through $G$, however, and so there is no smooth solution to the camber line problem for this shape. Slightly rounding the corner at $C$ does not change this fact, of more later.

Sufficient rounding can give rise to a problem with a smooth solution. Consider the airfoil shape shown in Figure 3. $A F$ is the bisector of the angle at $A$ and $B C$ is a circular arc centered at $D$. Even though the upper surface is not smooth at $B$ and $C$, there is a solution to the camber line problem with continuous first derivatives consisting of the bisector $A F$, the bisector of the angle at $E$ that lies within $C D E$, and a circular arc centered at $D$ that joins the two.

These solutions for camber lines rely on geometric constructions. The camber line problem can be cast into an analytical DAE problem as well to allow the use of analytical and numerical methods.

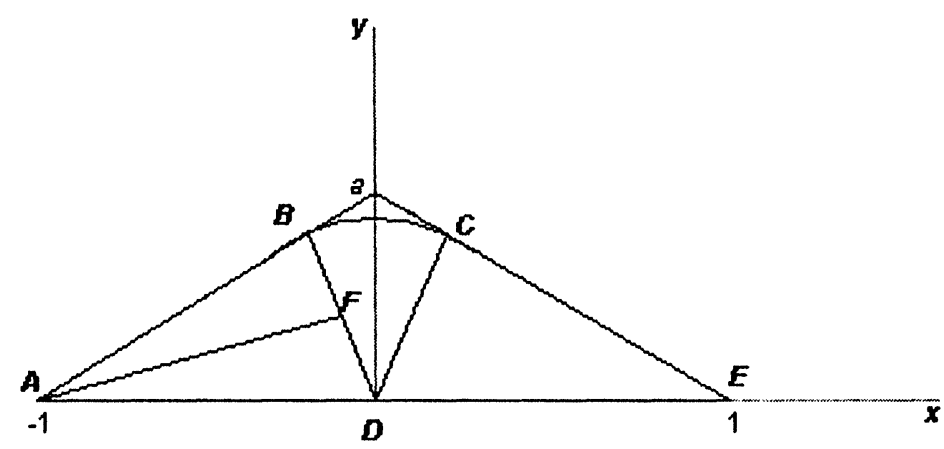

FIGURE 3 A triangular airfoil with top corner rounded by a circular arc. 


\section{FORMULATION OF THE INVERSION PROBLEM}

Say we are presented with a description of the upper and lower surfaces in the form

$$
\begin{aligned}
y_{u}\left(x_{u}\right) & =f\left(x_{u}\right) \\
y_{l}\left(x_{l}\right) & =g\left(x_{l}\right)
\end{aligned}
$$

where the functions $f$ and $g$ are known functions and we wish to construct a camber line and thickness function for the shape. Here we explicitly use the variables $x_{u}$ and $x_{l}$ to emphasize that they are to-bedetermined functions of $x$ which is measured along the chord of the airfoil. Now imagine that the point $\left(x, y_{c}\right)$ is a point on the camber line. We will not restrict attention to regions that look like practical airfoils, but instead consider regions of any shape with boundaries formed by two continuous functions.

Since it is on the camber line, the following equality must hold:

$$
y_{u}(x+\Delta x)-y_{c}(x)=y_{c}(x)-y_{l}(x-\Delta x)
$$

for some $\Delta x$ (see Fig. 4). From this the slope of the camber line is found to be:

$$
\frac{\mathrm{d} y_{c}}{\mathrm{~d} x}=\frac{-\Delta x}{y_{u}(x+\Delta x)-y_{c}(x)}
$$

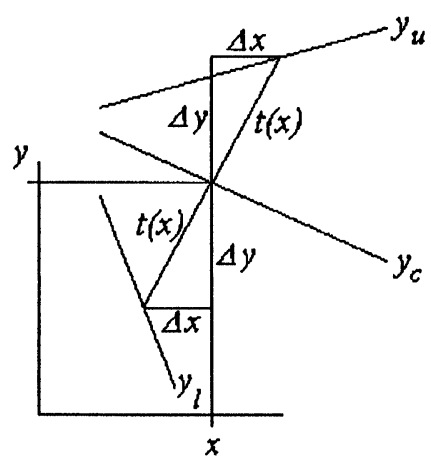

FIGURE 4 Definition sketch. The camber line and thickness functions are specified as functions of chord position, $x$. 
where $\Delta x$ is a function of $x$ and $y_{c}$ given by the equality above. $\Delta x$ is often a multivalued function of $x$ and $y_{c}$, even in very simple situations like we have seen earlier. In principle any point on the camber line can be used as an initial condition for solving the differential equation as long as there is a solution for $\Delta x$ at that point.

The problem posed is of the form

$$
\begin{aligned}
\frac{\mathrm{d} y}{\mathrm{~d} x} & =f(x, y, \Delta x) \\
g(x, y, \Delta x) & =0
\end{aligned}
$$

which is a semi-explicit nonlinear differential algebraic equation (DAE) [1].

Determination of the thickness function poses no additional difficulties and is given by:

$$
y_{t}(x)=t(x)=\sqrt{\Delta x^{2}+\left(y_{u}(x+\Delta x)-y_{c}(x)\right)^{2}} .
$$

\section{STRAIGHT LINE EXAMPLES}

\section{Linear Surfaces-Non-parallel}

The case with straight surfaces is one that can be analyzed exactly to a large degree, approximates conditions in a thin airfoil at least locally, and demonstrates some very important properties of the solution.

Consider the case where both the upper and lower surfaces are straight, non-parallel lines. Without loss of generality, one can move the point of intersection to the origin by simple shifts of coordinate. We will define the upper and lower surfaces by

$$
y_{u}=m_{1} \cdot x_{u}, \quad y_{l}=m_{2} \cdot x_{l}
$$

where

$$
x_{u}=x+\Delta x, \quad x_{l}=x-\Delta x
$$


Here we can solve for $\Delta x$ exactly:

$$
\Delta x=\frac{2 \cdot y_{c}(x)-\left(m_{1}+m_{2}\right) \cdot x}{\left(m_{1}-m_{2}\right)}
$$

which gives rise to the differential equation

$$
\frac{\mathrm{d} y_{c}}{\mathrm{~d} x}=\frac{2 \cdot y_{c}(x)-\left(m_{1}+m_{2}\right) \cdot x}{2 \cdot m_{1} \cdot m_{2} \cdot x-\left(m_{1}+m_{2}\right) \cdot y_{c}(x)} .
$$

This differential equation admits exact general solutions in implicit form after the substitution $v=x y_{c}(x)$. Explicit solutions can be obtained if $m_{1} m_{2}=-1$, that is the lines are perpendicular, because in that case the equation becomes an exact differential. The resulting solutions are:

$$
y_{c}(x)=-\frac{2 \cdot m_{1} \cdot x}{m_{1}^{2}-1} \pm \sqrt{\left(\frac{2 \cdot m_{1} \cdot x}{m^{2}-1}\right)^{2}+x^{2}+y_{0}^{2}+\frac{4 \cdot x_{0} \cdot y_{0} \cdot m_{1}}{m_{1}^{2}-1}-x_{0}^{2}}
$$

The solution which bisects the angle is the one where

$$
y_{0}=x_{0} \cdot \tan \left(\frac{\pi}{4}-\tan ^{-1}\left(\frac{1}{m_{1}}\right)\right) .
$$

Notice that only this solution passes through the origin. All other solutions initially between the two lines diverge from the bisector as the origin is approached.

Explicit exact solutions that pass through the point $\left(x_{0}, y_{0}\right)$ can also be obtained for the case $m_{1}=-m_{2}$ where now the equation is separable. These solutions are:

$$
y_{c}(x)=y_{0} \cdot\left(\frac{x}{x_{0}}\right)^{-1 / m_{1}^{2}} .
$$

Here we have the solution $y_{c}(x)=0$ for $y_{0}=0$ and $x_{0} \neq 0$ and all other solutions initially between the two lines do not pass through the origin. 
The fact that there is only one solution that enters the origin is not an artifact of the special choices made here. It can be shown either through inspection of the implicit solution for the general case or by simple geometrical argument that there is only one such solution in general. This result has far reaching consequences for the existence of solutions, of more later.

\section{Linear Surfaces-Parallel}

Parallel surfaces have an obvious solution for the camber line, but the formulation of the mathematical problem is interesting. Without loss of generality we can look at the problem where

$$
\begin{aligned}
& y_{1}(x)=1, \\
& y_{2}(x)=-1 .
\end{aligned}
$$

All other problems can be converted to this by suitable rotations, stretches, and translations of the coordinate system.

We find that solutions for $\Delta x$ only exist if $y_{c}=0$ and then there are an infinite number of solutions. Furthermore, the value of the slope of the camber line can take on any value. Of course solution can only proceed if the zero slope is chosen otherwise the solution curve is forced off of the $y_{c}=0$ line and no solution is possible. Hence all points on the camber line are singular points of the inversion problem.

\section{TRIANGULAR SHAPES}

Let's take a closer look at triangular regions. Consider the region bounded by

$$
\begin{aligned}
& y_{u}(x)=\left\{\begin{array}{l}
x+1, \quad-1 \leqslant x<0 \\
-x+1, \quad 0 \leqslant x \leqslant 1, \\
\text { undefined, otherwise }
\end{array}\right. \\
& y_{l}(x)=\left\{\begin{array}{c}
0, \quad-1 \leqslant x \leqslant 1 \\
\text { undefined, otherwise. }
\end{array}\right.
\end{aligned}
$$


The constraint equation becomes

$$
\begin{array}{rll}
2 \cdot y_{c}-(x+\Delta x+1)=0 & \text { if } & -1<x+\Delta x<0 \\
\text { and } & -1<x-\Delta x<1 \\
2 \cdot y_{c}+(x+\Delta x-1)=0 & \text { if } & 0<x+\Delta x<1 \\
\text { and } & -1<x-\Delta x<1
\end{array}
$$

No solution otherwise.

For this triangular shape (see Fig. 2) we have the following properties of the solution(s) to the constraint equation: in region $A B D$ there is one solution corresponding to points on the upper surface $A C$ and points on the lower surface $A F$, in region $D E F$ there is one solution corresponding to points on the upper surface $C E$ and points on the lower surface $A F$, in region $B D E$ there are two solutions (one corresponding to $A C$ and one corresponding to $C F$ ), and in $B C E$ there are no solutions. The fact that the line segments $B D, B E$, and $D E$ form the boundaries of these regions can also be deduced from the behavior of the partial derivative of the constraint equation with respect to $\Delta x$, that is by going through the exercise of computing the index of the system.

Since our solution is required to go from $A$ to $F$, the solutions in regions $A B D$ and $D E F$ must be the bisectors of the angles formed by the surfaces at the points $A$ and $F$, respectively. Clearly there is no smooth solution curve that joins the bisectors.

Now consider the consequences of the behavior at the leading and trailing edges. Each one is at least locally a pair of intersecting lines and hence there is a single solution curve in the neighborhood of the leading and trailing edges. As long as the local solutions for $\Delta x$ are distinct, smoothness of the solution requires that the local solution can be determined from a local linearization of the upper and lower surfaces about the points corresponding to the camber line position, i.e. if discrete, multiple $\Delta x$ 's are possible the one which varies only incrementally from the previously used one is allowed as the others will cause a discontinuity in slope for the camber line solution. This gives rise to unique solution curves coming out of both the leading and trailing edges until a point is reached where the upper and lower 
surfaces become parallel to the camber line (distinction solutions merge) or the camber line solution curves meet. If the solution curves meet where the upper and lower surfaces are parallel to both camber line solutions, then there is a smooth solution. Therefore a necessary condition on a smooth solution is that it must pass through a point where the upper and lower surfaces are parallel to the camber line before the solutions cross. Assuming that the point $C$ in the triangle example just given can be considered a place where the slope is zero, which is fully justified if that apex is slightly rounded in a very small neighborhood of $C$, then the line $B E$ is the locus of points where a camber line could exist that is parallel to both the upper and lower surfaces. We will call a curve with this property the parallel surfaces camber line locus. The solutions leaving $A$ and $F$, which are the bisectors, cross before reaching $B E$ and hence there can be no smooth solution to the problem.

The smooth solution curve cannot contact just any point on the parallel surfaces camber line locus for a smooth solution. An even more stringent necessary condition arises when the surfaces are locally parabolic, namely that if the camber line solutions join smoothly at that point they must also be tangent to the parallel surfaces camber line locus. It is not difficult to show that the parallel surface camber line locus is also a curve that separates regions where the camber line can exist within the shape from regions where it cannot if the appropriate parts of the upper and lower surfaces can be approximated with parabolas. (See Section 7 for details.) That means that as the solutions approach the parallel surfaces camber line locus from each direction they must be on the same side and so the only way to smoothly join the two curves is if they become tangent to the parallel surfaces camber line locus. That can only happen at points where the normal to the camber line is also normal to the parallel surfaces camber line locus. In this example the only point that meets this criteria is the point $G$ which does not lie on either bisector.

Now let us reconsider the case where there is a finite curvature of the upper corner of the triangle (see Fig. 5).

Here the altitude of the triangle is $a$ and once the upper corner is rounded the height becomes $b$. The point $F$ is the only point on the parallel surfaces camber line locus where its slope matches the slope of 


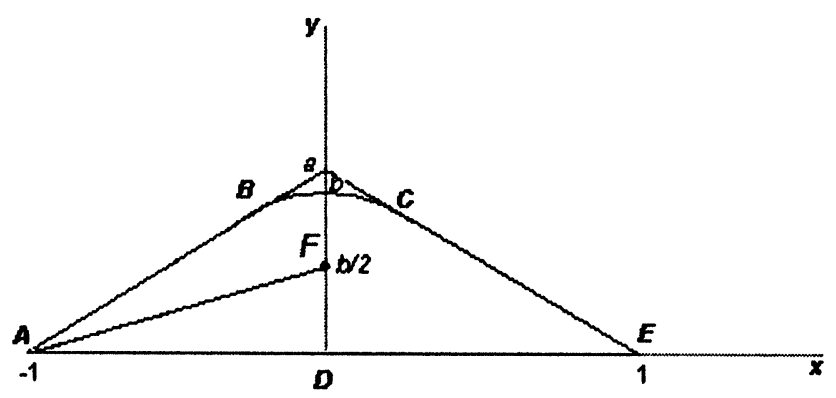

FIGURE 5 Triangular airfoil with rounded apex.

the surfaces and hence any smooth solution must go through $F$. If the line joining $A$ to $F$ lies above the bisector of angle $B A D$, then the camber line solution starting at $A$ cannot reach $F$. Hence a necessary (but not a sufficient) condition that a smooth camber line solution exists for this problem is that

$$
b \leqslant 2 \cdot \sqrt{\frac{\sqrt{a^{2}+1}-1}{\sqrt{a^{2}+1}+1} .}
$$

A sharper bound probably exists.

\section{PARALLEL SURFACES CAMBER LINE LOCUS}

The role of the parallel surfaces camber line locus and in particular the point where its slope matches the slope of the corresponding upper and lower surfaces plays a crucial role as described earlier. The locus of camber line positions where the upper and lower surfaces are parallel to the camber line can be written as the collection of points

$$
\left(x_{l}+\Delta x, \frac{\left(y_{u}\left(x_{l}+2 \cdot \Delta x\right)+y_{l}\left(x_{l}\right)\right)}{2}\right)
$$


subject to the condition

$$
\frac{\mathrm{d} y_{u}}{\mathrm{~d} x_{u}}\left(x_{l}+2 \cdot \Delta x\right)=\frac{\mathrm{d} y_{l}}{\mathrm{~d} x_{l}}\left(x_{l}\right) .
$$

In general this locus and any points where the slope of the locus matched the associated surface slopes should be identified first before attempting to calculate solutions numerically. Any solution curve that passes through this locus switches from a region where the upper and lower surfaces are diverging to one where they are converging. Solution curves in the regions where the upper and lower surfaces converge are unstable as described in Section 3 making numerical solution impossible.

\section{SEMICIRCULAR SHAPES}

Now consider the semicircular shape (see Fig. 6):

$$
\begin{aligned}
& y_{u}(x)=\left\{\begin{array}{l}
\sqrt{1-x^{2}}, \quad-1 \leqslant x \leqslant 1 \\
\text { undefined, otherwise }
\end{array}\right. \\
& y_{l}(x)=\left\{\begin{array}{c}
0, \quad-1 \leqslant x \leqslant 1 \\
\text { undefined, otherwise }
\end{array}\right.
\end{aligned}
$$

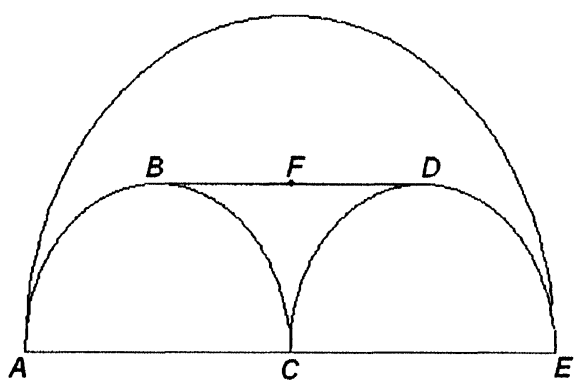

FIGURE 6 Semicircular airfoil. The large semicircle is the airfoil shape. 
which leads to the constraint equation

$$
\begin{array}{r}
2 \cdot y_{c}-\sqrt{1-(x+\Delta x)^{2}}=0 \text { if }-1<x+\Delta x<1 \\
\text { and }-1<x-\Delta x<1
\end{array}
$$

No solution otherwise.

In the semicircular regions $A B C$ and $C D E$ there is only one solution for $\triangle x$, in $B C D$ there are two solutions and above the curve $A B D E$ there are no solutions. The line segment $B D$ is the parallel surfaces camber line locus. The solution must pass through the point $F$ as it is the only point on the parallel surfaces camber line locus where the slope matches the slope of the corresponding camber line.

The camber line solution starting at $A$ solves the differential equation and initial condition

$$
\frac{\mathrm{d} y_{c}}{\mathrm{~d} x}=\frac{x+\sqrt{1-4 \cdot y_{c}^{2}(x)}}{y_{c}(x)}, \quad y_{c}(-1)=0 .
$$

The substitution

$$
w(x)=\frac{\sqrt{1-4 \cdot y_{c}^{2}(x)}}{x}
$$

leads to the separable differential equation

$$
x \cdot \frac{\mathrm{d} w}{\mathrm{~d} x}=\frac{-4-w(x)-4}{w(x)}
$$

which can be integrated exactly. The solution for $x$ and $y$ for $x$ between -1 and 0 can be expressed parametrically as

$$
\begin{aligned}
& x(w)=\frac{-e^{2-2 / w+2}}{w+2}, \\
& y(w)=\frac{\sqrt{1-x^{2}(w) \cdot w^{2}}}{2} \\
& -1 \geqslant w>-2 .
\end{aligned}
$$




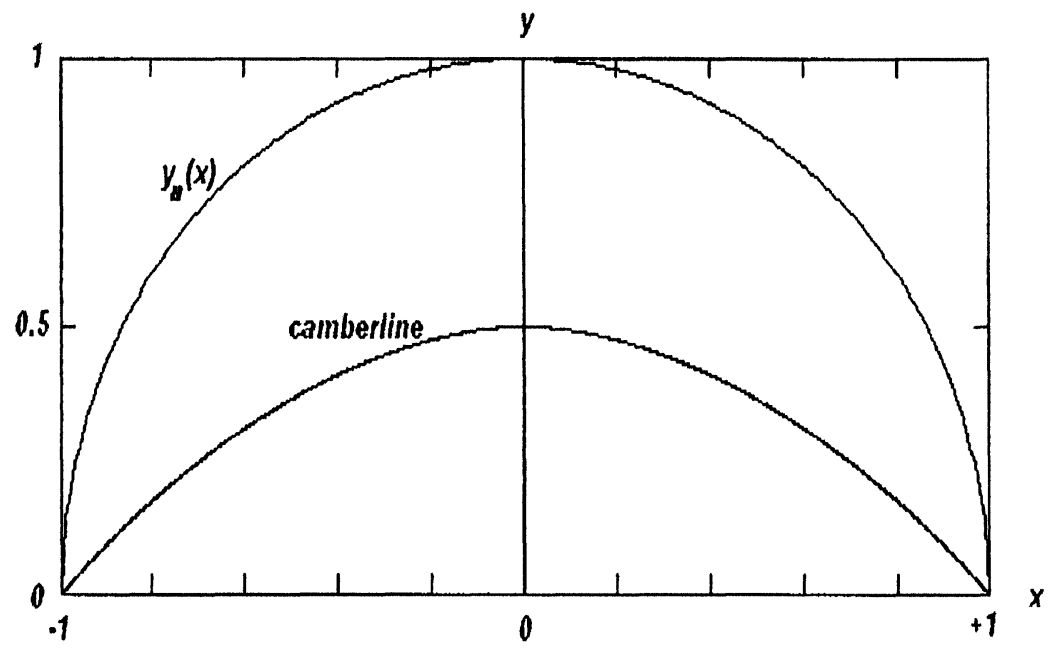

FIGURE 7 Camber line solution for the semicircular airfoil.

The solution from $x=0$ to $x=1$ can be found by symmetry and the total solution is shown in Figure 7.

\section{PARABOLIC SURFACES}

Here we will look at shapes where the upper and lower surfaces are parabolas.

\section{Symmetric Parabolas}

The camber line solution for a symmetric airfoil is trivial, but the structure of the mathematical problem has some interest. Here we will look at one example that can be readily extended to the general case of symmetric airfoils with parabolic surfaces.

Consider two curves given by

$$
\begin{aligned}
y_{u}(x) & =1-x^{2} \\
y_{l}(x) & =x^{2}-1
\end{aligned}
$$


which gives

$$
\Delta x=\frac{-y_{c}(x)}{2 \cdot x}
$$

If we restrict the surfaces to be defined in the interval $[-1,1]$ only, then solutions for $\Delta x$ exist in the region where

$$
\left|y_{c}\right| \leqslant\left\{\begin{aligned}
-2 \cdot\left(x+x^{2}\right), & -1 \leqslant x \leqslant 0 \\
2 \cdot\left(x-x^{2}\right), & 0<x \leqslant 1
\end{aligned}\right.
$$

The resulting differential equation for the camber line is:

$$
\frac{\mathrm{d} y_{c}}{\mathrm{~d} x}=\frac{2 \cdot x \cdot y_{c}(x)}{4 \cdot x^{2}-4 \cdot x^{4}-y_{c}^{2}(x)}
$$

The boundary conditions on this first order differential equation are:

$$
y_{c}(-1)=0, \quad y_{c}(+1)=0 .
$$

The obvious exact solution to this problem is $y_{c}=0$ which means that the solution must pass through the singularity at the origin. In the neighborhood of the singularity at the origin, where $4 x^{4}$ is negligible compared to the other terms, the solutions are

$$
y_{c}(x) \approx \pm \sqrt{K-\sqrt{K^{2}-4 \cdot K \cdot x^{2}}}
$$

which has a slope of \pm 2 if $K \neq 0$ and 0 if $K=0$. (This form of the solution is found by first writing $x$ as a function of $y_{c}$ and then treating the problem as a first order equation of homogeneous type. The resulting solution can then be inverted by solving a quadratic in $y_{c}^{2}$.) Given that the boundary on the region where there are solutions for $\Delta x$ has slope \pm 2 as the origin is approached, we see that all the solutions other than the line $y_{c}(x)=0$ that can pass through the singularity are attracted to the boundary of the region where camber lines may exist. Now imagine that this problem is being solved numerically. A small error will cause the solution to enter the singular point with slope of \pm 2 but there are an infinite number of solutions 
which leave the singular point with exactly the same slope. This type of behavior will occur whenever the camber line becomes parallel to both the upper and lower surfaces and the second derivatives of the upper and lower surfaces are equal in magnitude and of opposite sign.

Now consider an airfoil shape where there are multiple locations with the singular behavior exhibited at the origin in this example. Now it is possible to join the singular points both smoothly and non-uniquely giving rise to non-unique solutions to the camber line problem.

In this particular case the parallel surfaces camber line locus reduces to a single point which is the origin.

\section{Non-symmetric Parabolas}

The non-symmetric parabola case is important as it highlights the behavior of the solution in the vicinity of the point on the parallel surfaces camber line locus where the slope of the camber line matches the slope of the surfaces.

Consider the case of non-symmetric (with respect to the $x$-axis) parabolas:

$$
\begin{aligned}
& y_{u}(x)=a \cdot\left(1-x^{2}\right) \\
& y_{l}(x)=b \cdot\left(1-x^{2}\right)
\end{aligned}
$$

where $a \neq-b$ and $a>b$.

In this case there are two solutions for $\Delta x$ given by

$$
\Delta x=\frac{b-a}{a+b} \cdot x \pm \sqrt{x^{2} \cdot\left[\left(\frac{a-b}{a+b}\right)^{2}-1\right]+1-\frac{2 \cdot y_{c}}{a+b}}
$$

The curve where the radicand vanishes separates regions with solutions for $\Delta x$ from regions where there are no solutions. It is also the locus of points for the parallel surfaces camber line locus. In order for the camber line to go smoothly from $(-1,0)$ to $(1,0)$, the solution must pass through $(0,(a+b) / 2)$. An example with $a=2$ and $b=1$ is shown in Figure 8. The curve terminates at $A$ and $B$ because continuing the curve beyond those points requires continuation of the lower surface 


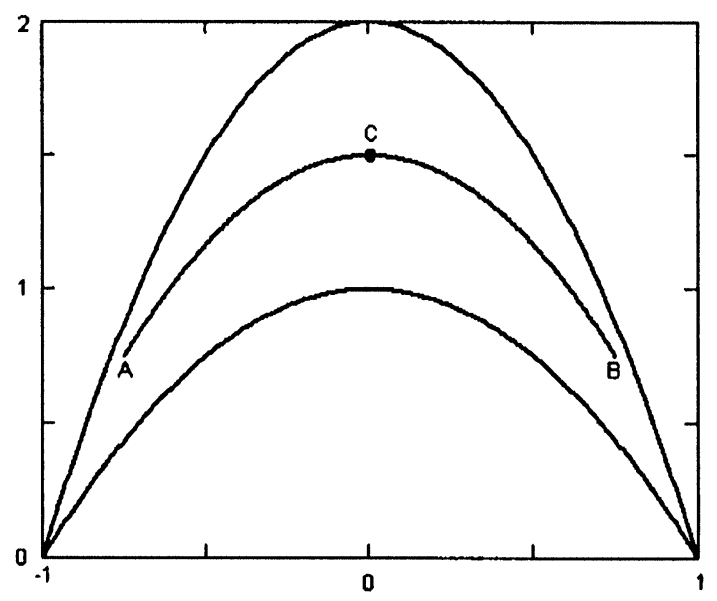

FIGURE 8 Parabolic upper and lower surfaces with parallel surfaces camber line locus $A C B$.

outside the range of definition for the lower surface. The fact that the parallel surfaces camber line locus is also a curve that separates regions where solutions exist is not surprising given that if two solutions for $\Delta x$ merge, it must happen at a place where the surfaces become parallel.

The camber line solution can be found numerically, but such solutions must be started at each end and joined at point $C$. Numerical integration beyond point $C$ in either direction is doomed to failure for the reasons given earlier.

Now we are in a position to discuss uniqueness of solutions. If there is only one point on the parallel surfaces camber line locus where the camber line would be parallel to the surfaces and its behavior is of the nonsymmetric parabola type, and if there is only one solution for $\Delta x$ in the neighborhood of both the leading and trailing edges, and if multiple solutions are distinct (with the exception of the parallel surfaces camber line locus where solutions can merge), then if a smooth solution exists it is unique. Having a single solution for $\Delta x$ at the leading and trailing edges guarantees that a unique solution leaves both the leading and trailing edges along the local bisectors. Distinct solutions for $\Delta x$ requires that any smooth solutions can only use incremental changes in the solution for $\Delta x$ and so the uniqueness is still maintained. Having a single point on the parallel surfaces camber line 
locus where the solution can pass requires that the solutions from either end must meet at that single point and each solution is unique from their respective ends to the parallel surfaces camber line locus. We therefore know that the solution for the semi-circular airfoil found in Section 5 is the unique solution to that problem. Most commonly encountered airfoil shapes would also satisfy these requirements.

\section{CONCLUDING REMARKS}

Here we have looked at a system of differential-algebraic equations (DAE) derived from a physical problem that is subject to boundary conditions. DAEs have been employed extensively in the study of constrained rigid body dynamics or chemically reacting systems where initial conditions are usually encountered instead..

Some important properties of the solution to this particular problem have been demonstrated. In particular, the intrinsic instability of the solution when integration is performed in the direction of converging surfaces shows that any numerical approach to this problem cannot start at one end and march across the domain. Also the a priori identification of points within the airfoil shape through which the solution must pass is of great utility in the discussion of existence and uniqueness of solutions. Furthermore, such points must be locations where numerical integrations must terminate since they mark locations where the surfaces change from diverging to converging as this is where the stability of the solution changes. Finally, simple smooth shapes that have no smooth camber line solution and a method for constructing smooth shapes with multiple camber line solutions have been identified.

\section{References}

[1] Brenan, K.E., Campbell, S.L. and Petzold, L.R. (1996) Numerical Solution of Initial-value Problems in Differential-algebraic Equations (SIAM, Philadelphia, PA).

[2] Munk, Max M. (1925) Elements of the Wing Section Theory and of the Wing Theory, NACA Report No. 191.

[3] Abbott, 1.H. and Doenhoff, A.E. (1949) Theory of Wing Sections (Dover, New York)

[4] Jacobs, E.N., Ward, K.E. and Pinkerton, R.M. (1932) The Characteristics of 78 Related Airfoil Sections from Tests in the Variable-density Wind Tumnel, NACA Report No. 460. 


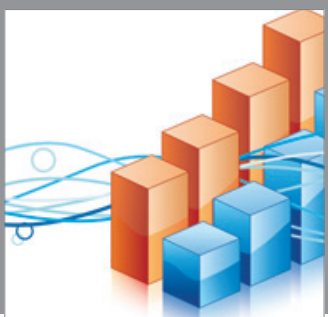

Advances in

Operations Research

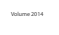

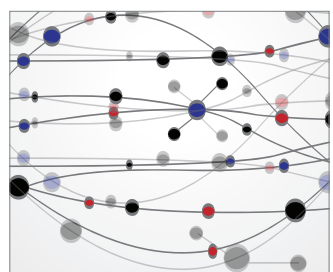

\section{The Scientific} World Journal
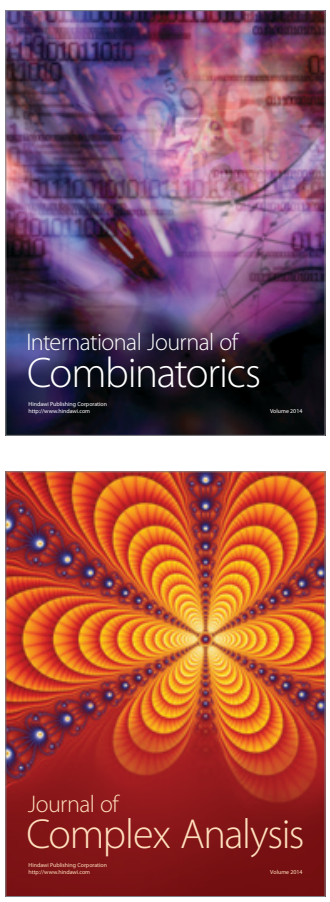

International Journal of

Mathematics and

Mathematical

Sciences
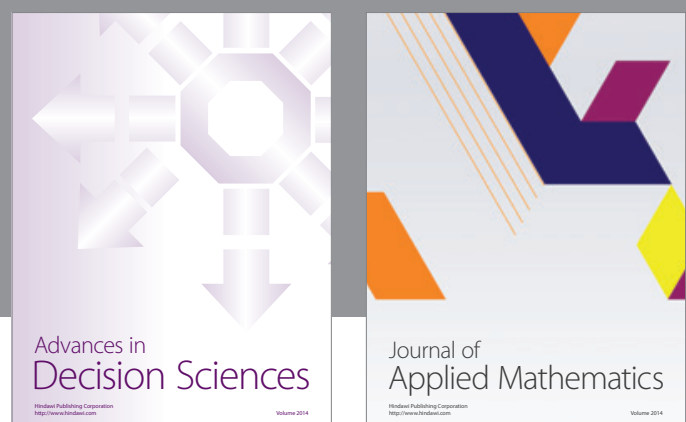

Journal of

Applied Mathematics
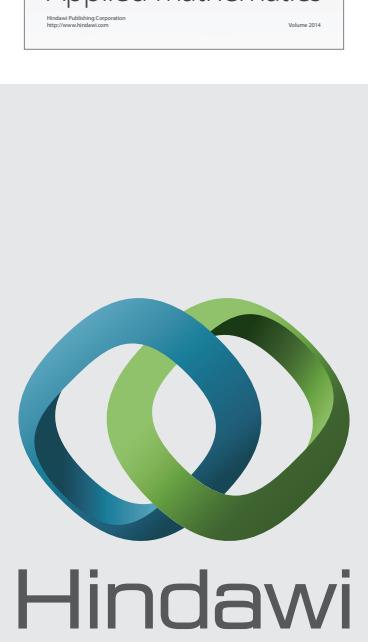

Submit your manuscripts at http://www.hindawi.com
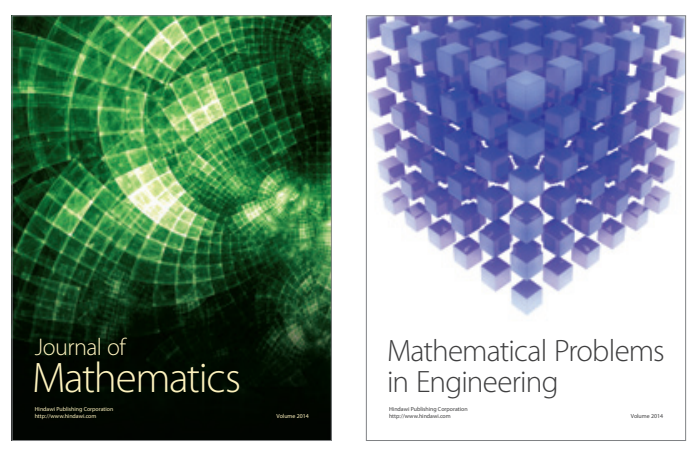

Mathematical Problems in Engineering
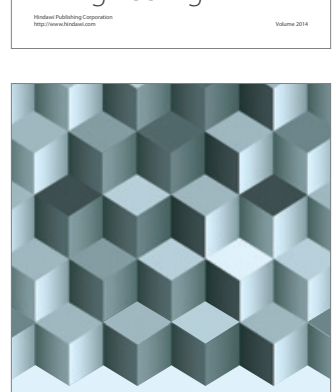

Journal of

Function Spaces
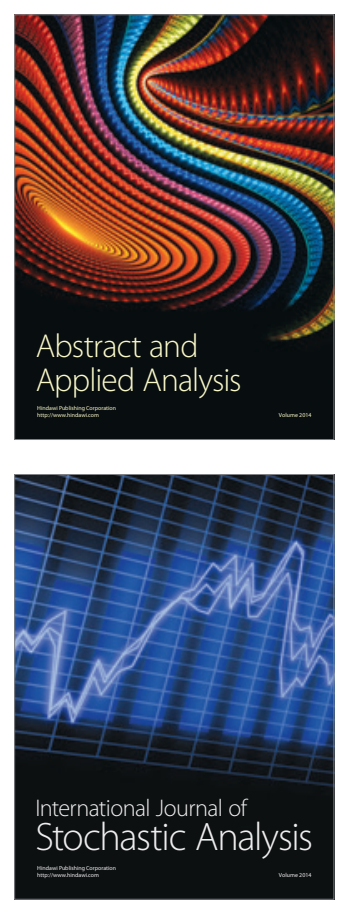

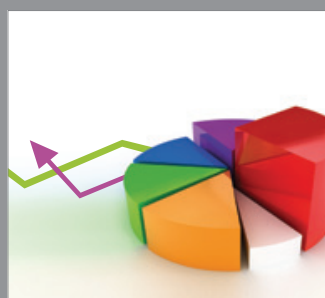

ournal of

Probability and Statistics

Promensencen
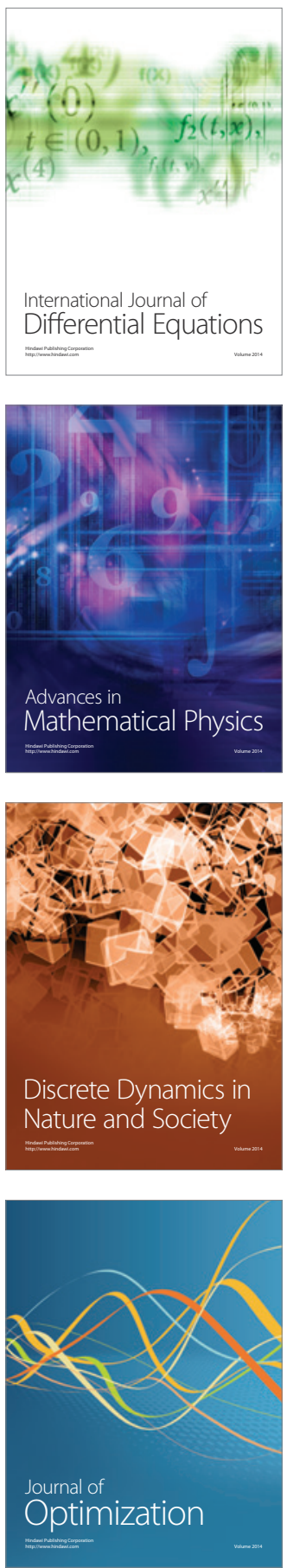